\title{
Patient Satisfaction with a Pilot Chronic Pain Management Programme in Cape Town, South Africa
}

\begin{abstract}
:
Background: The goals of a chronic pain management clinic include increasing patient knowledge about pain, developing pain management skills and increasing patients' confidence in their pain management abilities.

A Chronic Pain Management Programme (CPMP) based on evidence based guidelines was developed at a chronic pain management clinic to facilitate patient discharge to a primary healthcare level.

Aim: The aim of this study was to explore patient satisfaction with, acceptability of and the perceived success which could be due to the CPMP developed at the Chronic Pain Management Clinic of Groote Schuur Hospital, Cape Town.

Methods: Patients ( $n=14)$ were referred to the pilot study from the Chronic Pain Management Clinic. As a pilot, four courses were run over a period of one year. In order to reach the research aim, an eleven-question, structured

\begin{tabular}{c} 
Parker R, \\
BSc(Phys), \\
BSc(Med)(Hons)Ex.Sci(Phys), \\
MSc(Pain)'; \\
Burgess S, BSc(Phys); \\
Dubaniewicz A, BSc(Phys); \\
Gouws L, BSc(Phys); \\
Krone J, BSc(Phys); \\
Madden V, BSc(Phys); \\
Nortje C, BSc(Phys); \\
Parsons C, BSc(Phys); \\
' University of Cape Town, South Africa. \\
\hline
\end{tabular}
open-ended interview was conducted with all participants.

Results: Fourteen patients enrolled in the CPMP. Responses were favourable with participants emphasising the role of increased knowledge about pain, the role of exercise and of stress management techniques. Participants also recognised a positive change in behaviours and attitudes following participation in the CPMP.

Conclusions: Findings suggest that participants found the format of the course acceptable as regards course content, structure and delivery. Participant responses suggest that the course was acceptable and perceived as useful. However, future courses would benefit from refresher courses or structured support groups.
\end{abstract}

\section{KEYWORDS: CHRONIC PAIN, PATIENT EDUCATION, PATIENT SATISFACTION.}

\section{INTRODUCTION}

Chronic pain has been variously described as pain lasting for more than three months (Merskey and Bogduk, 2002); pain which has outlived its usefulness (Gifford, 1998) or pain which continues despite the original pathology having resolved (Main and Spanswick, 2000). Chronic pain has been reported to affect one in five people worldwide. The effects of chronic pain are multiple with one in three people with chronic pain being unable or less able to maintain an independent lifestyle. In addition, people living with chronic pain report that their condition causes strain in or, breaks one in four close relationships (International Federation for Medical \& Biological Engineering, 2006).

The current recommended approach to the treatment of chronic pain is born from the understanding that true chronic pain will never disappear completely
(Turk and McCarberg, 2005). Treatment veers away from a curative approach in favour of one that aims to equip patients to manage their pain in the long term. Guidelines published by the International Association for the Study of Pain (IASP) recommend that chronic pain be managed through interdisciplinary clinics which include physicians, physiotherapists, occupational therapists, psychiatrists and nurses with specialised training in pain management (Main and Spanswick, 2000).

The chronic pain management approach has been implemented in interdisciplinary clinics as a means of improving physical functioning, selfefficacy, quality of life, psychological elements and behaviour in people with chronic pain. The goals of CPMP are to maximise patients' knowledge about pain, develop pain management skills and to increase confidence in their own abilities to be active participants in the management of their pain (Morley and Eccleston, 2008). This pain management approach has been reported to minimise the impact of chronic pain on the patient by decreasing disability, decreasing dependency on medication and empowering people with pain to continue being active contributors to

\section{Correspondence to:}

Romy Parker

Division of Physiotherapy,

School of Health and

Rehabilitation Sciences,

University of Cape Town

F56 Old Main Building,

Groote Schuur Hospital,

Observatory 7925, South Africa

Tel: (021) 406-6571(w)

Fax: (021)406-6323(f)

Email: Romy.parker@uct.ac.za 
society (Chodosh et al., 2005, Harper, 2002, Lorig et al., 2001, Von Korff et al., 2005, Yip et al., 2007).

In order to maximise effectiveness, a cognitive behavioural approach aimed at increasing knowledge and achieving a change in behaviour through the use of education, problem solving, activity management (exercise and pacing) and skill acquisition (e.g. relaxation and stress management) must be adopted (Morley et al., 2008). This type of approach is based on the assumptions that patients can learn general principles and skills which will help them cope with their pain; that patients should be active participants in their own care and that improved self-care skills can improve quality of life (Bandura, 1997).

There is good evidence to support the use of CPMP (Level I and II evidence according to the Oxford Bandolier system) in developed societies (Flor et al., 1992, Turk and McCarberg, 2005). However, there is a paucity of literature describing the use of CPMP in developing countries faced with the challenges of limited healthcare budgets, human resources and social and cultural diversity.

The aim of this study was to investigate patient satisfaction with, acceptability of and the perceived success of a CPMP developed by the chronic pain management clinic of Groote Schuur Hospital. Participants' perceptions regarding successful and unsuccessful components of the programme, as well as attitude and behaviour changes which they experienced as a result of taking part in the CPMP, were explored. This paper reports on the qualitative responses of the participants of the pilot study of the CPMP which contribute to an understanding of how well the CPMP met its goals of increasing participants' knowledge of pain, pain management skills and confidence about coping with their pain.

\section{METHOD}

\section{Setting}

In 2006 it was recognised that the pain management clinic at Groote Schuur Hospital was struggling to cope with a growing number of patients. The multidisciplinary team felt that many of these patients had the potential to be managed at a primary healthcare level but lacked the knowledge and skills required for this. Recognising that the goals of chronic pain management programmes include decreasing the impact of pain on the individual by increasing active participation in the management of their condition through raising levels of selfefficacy (Main and Spanswick, 2000), a Chronic Pain Management Programme (CPMP) was developed.

The CPMP was designed to be run on an outpatient basis in the Physiotherapy department of Groote Schuur Hospital. The venue was selected on the basis of suitability, availability and affordability. The aim of the programme was to increase self-management, engaging patients as active participants in managing their health. This would enable discharge from the tertiary services of the chronic pain management clinic to a primary healthcare level.

\section{Sample}

Over the period of one year, fourteen (14) patients were referred to the CPMP following assessment by the multidisciplinary team of the Chronic Pain Management Clinic (psychiatrist, physiotherapist and a pain specialist consultant). Patients referred to the programme were informed that they would be asked to commit to attending a course weekly for a six week period to facilitate their discharge to a primary care clinic. It was explained to all potential participants that the programme would require them to exercise and active participation was encouraged. As a pilot, four courses were run over a period of one year.

Thirteen of the patients who participated in the pilot of the CPMP were contacted and invited to participate in qualitative interviews to evaluate the programme. One participant could not be traced. One participant was not available for interview due to unrelated health problems. Twelve of the participants gave informed consent and participated in the interview $(n=12)$.

\section{Procedure}

A pre-experimental cross-sectional pilot study was conducted. The project was approved by the Faculty of Health Sciences Ethics Committee of the Univer- sity of Cape Town and the Groote Schuur Hospital Medical Superintendent.

All patients were provided with an information sheet and were given the opportunity to ask questions prior to signing informed consent. The student conducting the interviews emphasised that she had not been involved in the programme and that although the name of the physiotherapist who had conducted the programme was on the information sheet, all information would be strictly anonymous. Data for each participant were encoded to ensure anonymity and participants could withdraw at any time with no effect on their future management.

A multidisciplinary management approach which has been successfully implemented for the management of chronic pain disorders was used as a guideline for the development of a six week outpatient CPMP (Flor et al., 1992, Main and Spanswick, 2000, Moore et al., 2000, Olason, 2004, Ostelo et al., 2005). Studies on the efficacy of CPMP have investigated various models of delivery. These include inpatient programmes ranging from 4-weeks (Williams et al., 1993) to 7-weeks in duration (Olason, 2004), combination inpatient/outpatient programmes (Main and Spanswick, 2000) and six to eight week outpatient programmes (Moore et al., 2000). The CPMP at Groote Schuur Hospital was held once a week for an hour and half over a six week period, a total of nine hours. This was the minimum time period recommended by the literature and was influenced by the challenges of clinical staff and venue availability, and affordability for patients with regard to transport and treatment costs. Each session included a 20 minute supervised exercise session; a 50 minute education and discussion session followed by a 15 minute supervised relaxation period.

The participants in each CPMP included the facilitating physiotherapist, two final year physiotherapy students and two to seven patients. A "closed group" format was used to maximise confidentiality and group cohesion. All participants discussed and signed a "contract of attendance" in which confidentiality; timekeeping, attendance and 
programme content were agreed upon. With group consent, participants could invite a family member or partner.

The 20 minute exercise session was supervised by the physiotherapist. Supervised exercise sessions are a critical component of CPMP as supervision by an expert increases patient confidence and may improve adherence to an exercise programme (Fillipas et al., 2006). Exercises were selected with the aims of teaching practical skills of pacing and normalising movement to decrease kinesophobia. Patients were instructed to exercise at the "somewhat hard" intensity level as described on the Borg Scale of perceived exertion (Borg, 1982). Aerobic exercises included walking and step-ups and were performed in intervals alternating with stretching and strengthening exercises. The use of any specialised equipment was avoided in order to demonstrate that the exercises could be carried out in participants' homes.

A curriculum outline based on the literature was developed in order to ensure consistency (Main and Spanswick, 2000, Robb et al., 2006). Topics selected for discussion included: pain physiology and mechanisms and how these related to each person's personal pain experience; exercise and how to adapt an exercise programme for each person; how to use goal setting and pacing to tackle the challenges of daily life; recognising factors and situations which increase stress and how to use stress management techniques to manage these; eating a balanced diet including economical sources of food and methods of preparation; and why and how to use prescribed medication. Active participation was facilitated by the physiotherapist with sharing of personal experiences and insights being encouraged. Discussion sessions were concluded with problem solving leading to participants selecting personal goals relating to the topic discussed. Goals were agreed on by the group and feedback given weekly on goal achievement. Goal setting and problem solving of goals is a fundamental component of cognitive behavioural therapy aimed at integrating education into a change in behaviour (Bandura, 1997, Flor et al., 1992, Morley et al., 2008).

Relaxation sessions utilised a systematic relaxation technique (Payne, 2000). Visualisation methods of relaxation were avoided as participants included various cultural groups with differing first languages. These differences could result in different interpretation of visualisation images resulting in difficulty relaxing or even increasing stress levels (Schaffer and Yucha, 2004).

On completion of the six week programme all participants were provided with an information booklet summarising the topics discussed. The aim of the booklets was to include the basic curriculum as well as any added input from the participants. In this way each group received an individualised booklet.

Prior to the follow-up interview, participants' medical folders were consulted to determine demographic data including age, gender, home language, diagnosis and occupation. One-on-one semi-structured interviews were conducted with patients who participated in the pilot CPMP in order to explore patient satisfaction with the programme, its acceptability to patients and its success in achieving a perceived change in behavior.

An eleven-question, structured openended interview was conducted. The interview questions (Table 1) aimed to elicit information about specific elements of the programme while allowing open-ended elicitation of participants' experiences. Experienced pain management clinicians reviewed the questionnaires in order to establish face validity. The questions were felt to adequately explore focus areas of the CPMP. The questionnaire was piloted on patients attending the outpatient physiotherapy department and terminology which was felt to be confusing was clarified.

Participants were interviewed by a final year physiotherapy student who had not participated in the programme. The student conducting the interviews was trained in interviewing techniques. Interviews took place in the department where the programme had been conducted. Hand notes of the interviews were made by the student conducting the interview and responses were read back to the participants to ensure dependability. Interviews took place between one month and one year after participation in the CPMP. Closed questions which elicited yes or no

\section{Table 1. Structured Questions used in the Participant Interviews}

\section{When did you attend the Chronic Pain Management Course? Did you attend the whole course?}

If not why did you stop attending the course?

2. Did attending the Chronic Pain Management Course help you have an understanding of you pain? How?

3. Has the Chronic Pain Management Course helped you to manage your everyday pain?

4. During the course what changed in your everyday life? Are you still managing to achieve these changes?

5. What did you find most interesting about the course?

6. What did you find most helpful from the programme that improved your pain?

7. What goals did you set for yourself during the programme?

8. Are you still managing to achieve these goals? If not, why not?

9. Were there any negative factors you found in the programme?

10. Did you benefit from participating in the programme?

11. Are you still in contact with other participants from the Chronic Pain Management Course? 
responses were analysed using descriptive statistics. Open questions which elicited qualitative data were post-coded into different themes which emerged during analysis.

\section{RESULTS}

Fourteen people participated in the CPMP, two males and twelve females. The median age was 48 years (range 34 - $59 \mathrm{yrs}$ ). The home language of seven of the participants was English, five Afrikaans and two IsiXhosa. The majority of patients were referred with a diagnosis of chronic low back pain (8), other diagnoses included fibromyalgia (2), arthritis (1), chronic headaches (1), chronic whiplash (1) and complex regional pain syndrome (1). Eight participants were recorded in their medical records as unemployed, four as housewives and two as employed. Three of the fourteen participants failed to complete the CPMP. All three dropped out after the second session (failed to attend from the third week). Five patients attended the first course, three the second, four in the third and only two patients in the last course of the year. Patients who took part in the first course were interviewed 12 months after completion of the course, those who participated in the second course were interviewed 10 months after completion, those on the third course were interviewed five months after completion and patients who participated in the final course were interviewed one month after completion.

When asked whether the CPMP had helped them to understand their pain, all 12 participants responded positively. Responses were grouped into two themes. Firstly, participants identified understanding the physiology of chronic pain as helpful in understanding their experiences, e.g. "understood my pain and what causes it". The second aspect which helped understanding of pain, was recognising that interventions focus on management and not treatment, with responses including: "to manage and deal with the pain"; "you have to learn to cope with your pain"; "cannot get rid of the pain but can learn to cope with it".

Nine participants responded positively to the question "Has the course helped you to manage your everyday life?" One responded that the course helped to change their life "sometimes" while the remaining participants felt that there had been no change.

Ten participants could identify particular areas in their lives which had improved during the course. These related directly to the topics presented in the course. Increased ability to perform physical tasks was identified by nine of the participants with responses indicating pacing skills and exercises/fitness facilitating this, e.g. "Can perform daily activities easier"; "Started to walk, do things for myself"; "Can do chores at home" and "More capable of doing things around the house". Relaxation and stress management skills were identified by six of the participants as helping them to cope with their pain: "Learned to relax and ease the pain"; "Less impatient and critical with myself, I didn't feel so alone and I realised that there would be good and bad times"; "I can relax now"; "I started to be more positive" and "I'm more motivated to do things, not as anxious anymore". Two participants reported that nothing had changed in their everyday lives.

Seven participants felt that they were still managing to maintain the changes which they had implemented during the course. Three participants responded that they could maintain the changes but that it was an effort saying "Yes, if I try"; "Yes, if I do what I learnt" and "Sometimes. It would be nice to do a refresher course".

Themes which arose regarding interesting aspects of the CPMP included learning about pain, managing pain, and exercise. Four participants said that every session was interesting; "I enjoyed every session so much that I couldn't wait to go back"; "I loved everything especially teaching about yourself and how to cope". With regard to what was most helpful in improving their pain, four participants said that exercise was most helpful; and four said that understanding their pain and pain mechanisms was most helpful. It would appear that all the topics presented were felt to be beneficial by the participants.

When asked about what goals they had set for themselves during the pro- gramme and whether they were maintaining these goals, all the participants could recall at least two goals they had set. The goals set by the participants fell into two categories: functional goals (e.g. exercise: "to take 20 minute walks") and psychosocial goals (e.g. "to give myself resting time"; "to aim to say no if I'm in too much pain"; "to do relaxation techniques every day"). This appears to be a reflection of pain limiting different areas in their lives and that the course assisted them in addressing several of the dimensions of chronic pain. Three participants reported that they were not able to achieve or maintain their goals due to their pain, saying: "I don't if I'm too tired or it's too painful"; "too tired and in too much pain" and "No, it's too stressful".

Ten participants, (including two who had only completed two sessions) reported benefiting from the CPMP. Another ten participants (three who had not completed the course) reported that there were no negative factors about the course. One person suggested that "there is not enough interaction with people and that the course needs a better support group". This is reinforced by only three participants reporting having kept in contact with other group members.

Three female participants failed to complete the full six week programme. All three were recorded as employed (a factory machinist, two housewives). One of these participants spoke Afrikaans as her home language, the others spoke English. All three attended only the first two sessions of different courses. These participants gave varying reasons as to why they failed to complete the CPMP. One participant reported that she was in too much pain and "I didn't think the course would help". The other participants indicated difficulty with attending the course with one having transport difficulties and the other reporting "I could not get time off work".

\section{DISCUSSION}

The strongest theme which emerged from the interviews was an overall positive response to the course with participants reporting increased levels of self-efficacy. Comments such as "learnt to say no"; "started to do things for 
myself and be more positive" and "more motivated to do things and I'm not as anxious anymore" all indicate an increased awareness of self-need and belief in one's abilities. The majority reported maintaining changes they had implemented during the course, some of these more than a year after completing the course suggesting long term behaviour change. These are similar findings to those reported in CPMP in developed countries with people reporting similar increasing awareness of one's own needs, increased levels of self-efficacy and improved communication abilities following participation in CPMP (Flor et al., 1992, Kralik et al., 2008, Morley et al., 2008, Ostelo et al., 2005). This is particularly encouraging considering that the pilot CPMP was run as an outpatient programme in the minimum number of hours recommended by the literature.

Participants' reporting that they "could not wait to come back" to the course appears to suggest that the topics were relevant and that participants were engaged in the process. Two participants indicated that further ongoing support would be useful, one asking for a support group and another requesting a follow up course. This could be addressed through a peer-led chronic pain support group who could arrange support meetings and refresher topics. This aspect of needing further support and "refresher" courses has been identified in other chronic pain management clinics (Kralik et al., 2008, Main and Spanswick, 2000, Williams et al., 1996) and suggests that CPMP should be structured to include follow-up / refresher courses and or referral to patient-led support groups.

Only two participants spoke IsiXhosa as their home language. This was unexpected as one third of the population of the Western Cape region which is serviced by the Chronic Pain Management Clinic is IsiXhosa speaking (Statistics South Africa, 2005). While these two participants responded positively to the course, language and cultural acceptability for IsiXhosa speakers cannot be presumed.

Early termination of participation in the programme appeared to be due to factors not directly related to the CPMP. Difficulty with negotiating time off work could be managed through employer education. Information about the course including expected outcomes of decreased absenteeism and increased productivity (Marks et al., 2005, Lorig et al., 2001, Taylor et al., 2006) to employers may facilitate patients being released from work to attend the CPMP. Alternatively offering a CPMP outside of working hours may facilitate participation by patients as well as family. Challenges surrounding transport are common in developing countries. This could be addressed by moving the CPMP from a tertiary institution to a primary healthcare level. This is being explored by the authors with physiotherapists receiving training to facilitate similar courses at community health care centres. The patient who failed to complete the programme due to high levels of pain may have been referred at an inappropriate stage. Patients should be referred for CPMP only once appropriate pharmacological management of pain has been instituted (Main and Spanswick, 2000). In order to facilitate referral to CPMPs at the appropriate time the interdisciplinary team including the pain specialist, psychiatrist and physiotherapist must assess the patient to evaluate whether they will be able to engage with a CPMP.

\section{LIMITATIONS}

The small sample size in this pilot study limits the statistical analysis and interpretation of the results. This is compounded by the small number of IsiXhosa speakers who participated in the programme, limiting application of the results to this population group. The pre-experimental design of this study further limits interpretation of the results; specifically, no causal relationship can be shown. A further study is recommended using a more rigorous quasi-experimental research design. While a true experimental design would be optimal to evaluate the effectiveness of a CPMP the heterogeneity of chronic pain patient groups and the requirement that the CPMP be tailored to the needs of the individual group mean that controlling all the variables is not possible. To further strengthen future research, the use of standardised outcome measures which have been validated for this population is recommended, as is a larger sample. Further qualitative research, perhaps using the participatory action research methodology would provide further insight into the experience of chronic pain and impact of the CPMP (Kralik et al., 2008).

Bias in the results cannot be excluded on two counts. The physiotherapist, who facilitated the CPMP, was also involved in the study and as a result her name was presented to participants on the informed consent form. Although the participants were assured of confidentiality it is possible that mention of the physiotherapist's name may have biased responses in favour of the programme. While the participants were reassured that the student collecting the data was not involved in the course and that responses would be anonymous, the use of the same venue in which the course was run for the interviews may have influenced responses.

\section{CONCLUSION}

Based on this pilot study, the CPMP appears to be a useful and acceptable programme for patients with chronic pain referred to the chronic pain management clinic of Groote Schuur Hospital. The positive feedback from patients who completed the CPMP suggests that patients were satisfied with the programme and that they found it acceptable. Patient responses indicate that the CPMP has equipped its participants with some behavioural skills which may facilitate their discharge to a primary care level. Participants' positive feedback regarding goals set during the course and ongoing use of these skills suggests that a change in levels of selfefficacy may have been achieved. All components of the programme were described as interesting and helpful by the participants. Quantitative analysis of the effectiveness of the CPMP in a quasi-experimental study needs to be conducted to further explore the efficacy of this approach with this population. In addition participatory action research would provide further insight into the experience of living with pain and the impact of a CPMP. 


\section{REFERENCES}

Bandura, A 1997 Self-Efficacy:The Exercise of Control. W.H. Freeman and Company, New York

Borg GA 1982 Psychophysical bases of perceived exertion. Medicine and Science in Sports and Exercise 14:377-81.

Chodosh J, Morton SC, Mojica W, Maglione M, Stuttorp MJ, Hilton L, Rhodes S \& Shekelle P 2005 Meta-Analysis: Chronic Disease SelfManagement Programs for Older Adults. Annals of Internal Medicine 143:427-438.

Fillipas S, Oldmeadow LB, Bailey MJ \& Cherry CL 2006 A six-month, supervised, aerobic and resistance exercise program improves self-efficacy in people with human immunodeficiency virus: a randomised controlled trial. Australian Journal of Physiotherapy 52:185-190.

Flor H, Fydrich T \& Turk DC 1992 Efficacy of multidisciplinary pain treatment centres: a meta-analytic review. Pain 49:221-30.

Gifford, L 1998 Pain, the Tissues and the Nervous System: A conceptual model. Physiotherapy 84:27-36.

Harper, B 2002 Improving physical function in chronic pain syndrome patients. In: Gifford, L (ed) Topical Issues in Pain 4. CNS Press, Falmouth

International Federation for Medical \& Biological Engineering 2006 World Health Organization Supports Global Effort to Relieve Chronic Pain. Medical \& Biological Engineering and Computing, 70.

Kralik D, Koch T \& Price K 2008 Qualitative research with people who live with chronic illness and pain. In: Wittink HM \& Carr D (Eds.) Pain Management: Evidence, Outcomes and Quality of Life. Elsevier, Edinburgh

Lorig KR, Ritter P, Stewart AL, Sobel DS, Brown BW, Bandura A, Gonzalez VM, Laurent DD \& Holman HR 2001 Chronic disease self-management program: 2-year health status and health care utilization outcomes. Medical Care 39:1217-23.

Main CJ \& Spanswick CC 2000 Pain Management: An Interdisciplinary Approach. Churchill Livingstone, London

Marks R, Allegrante JP \& Lorig K 2005 A review and synthesis of research evidence for self-efficacy-enhancing interventions for reducing chronic disability: implications for health education practice (part I). Health Promotion and Practice 6:37-43.
Merskey H \& Bogduk N 2002 Classification of Chronic Pain. IASP Press, Seattle

Moore JE, von Korff M, Cherkin D, Saunders K \& Lorig K (2000) A randomized trial of a cognitive-behavioral program for enhancing back pain self care in a primary care setting. Pain 88:145-53.

Morley S \& Eccleston C 2008 Cognitivebehaviour therapy for chronic pain in adults. In: Wilson PR, Watson PJ, Haythornthwaite JA \& Jensen, TS (eds) Chronic Pain 2nd Ed. Hodder Arnold, London

Morley S, Williams A \& Hussain S 2008 Estimating the clinical effectiveness of cognitive behavioural therapy in the clinic: evaluation of a CBT informed pain management programme. Pain 137:670-80.

Olason M 2004 Outcome of an interdisciplinary pain management program in a rehabilitation clinic. Work 22:9-15.

Ostelo RW, van Tulder MW, Vlaeyen JW, Linton SJ, Morley SJ \& Assendelft WJ 2005 Behavioural treatment for chronic low-back pain. Cochrane Database Systematic Reviews, CD002014.

Payne R 2000 Relaxation Techniques: a practical handbook for the health care professional. Churchill Livingstone, Edinburgh

Robb KA, Williams JE, Duvivier V \& Newh DJ 2006 A pain management program for chronic cancer-treatment-related pain: a preliminary study. The Journal of Pain 7:83-90.

Schaffer SD \& Yucha CB 2004 Relaxation \& pain management: the relaxation response can play a role in managing chronic and acute pain. The American Journal of Nursing 104:75-82

Statistics South Africa 2005 Data Census 2001: Primary tables Western Cape: Census '96 and 2001 compared. Government of South Africa, Pretoria.

Taylor WJ, Dean, SG \& Siegert RJ 2006 Differential association of general and health self-efficacy with disability, health-related quality of life and psychological distress from musculoskeletal pain in a cross-sectional general adult population survey. Pain 125:225-232.

Turk DC \& McCarberg B 2005 NonPharmacological Treatments for Chronic Pain: A disease management context. Disease Management and Health Outcomes 13:19-30. Von Korff M, Balserson BHK, Saunders K, Miglioretti DL, Lin EHB, Berry S, Moore JE
\& Turner JA 2005 A trial of an activating intervention for chronic back pain in primary care and physical therapy settings. Pain 113:323-330.

Williams A, Nicholas MK, Richardson PH, Pither CE, Justins DM, Chamberlain JH, Harding VR, Ralphs JA, Jones SC, Dieudonne I 1993 Evaluation of a cognitive behavioural programme for rehabilitating patients with chronic pain. British Journal of General Practice 43:513-8.

Williams A, Pither CE, Richardson $\mathrm{PH}$, Nicholas MK, Justins DM, Morley S, Diamond A, Linton S, Vlaeyen J, Niilges P \& Eccleston C 1996 The effects of cognitivebehavioural therapy in chronic pain. Pain 65:282-4.

Yip YB, Sit JW, Fung KKY, Wong DYS, Chong SYC, Chung LH \& Ng TP 2007 Impact of an arthritis self-management programme with an added exercise component for osteoarthritic knee sufferers on improving pain, functional outcomes and use of health care services: an experimental study. Patient Education and Counselling 65:113-121. 